\title{
X-chromosome-wide profiling of MSL-1 distribution and dosage compensation in Drosophila
}

\author{
Gaëlle Legube, ${ }^{1}$ Shannon K. McWeeney, ${ }^{2}$ Martin J. Lercher, ${ }^{1,3}$ and Asifa Akhtar ${ }^{1,4}$ \\ ${ }^{1}$ European Molecular Biology Laboratory, 69117 Heidelberg, Germany; ${ }^{2}$ Division of Biostatistics, Department \\ of Public Health and Preventative Medicine, Oregon Health and Science University, Portland, Oregon 97239, USA; \\ ${ }^{3}$ Department of Biology and Biochemistry, University of Bath, Bath BA2 7AY, United Kingdom
}

In Drosophila, dosage compensation is achieved by a twofold up-regulation of the male X-linked genes and requires the association of the male-specific lethal complex (MSL) on the $\mathrm{X}$ chromosome. How the MSL complex is targeted to $\mathrm{X}$-linked genes and whether its recruitment at a local level is necessary and sufficient to ensure dosage compensation remain poorly understood. Here we report the MSL-1-binding profile along the male $\mathbf{X}$ chromosome in embryos and male salivary glands isolated from third instar larvae using chromatin immunoprecipitation (ChIP) coupled with DNA microarray (ChIP-chip). This analysis has revealed that majority of the MSL-1 targets are primarily expressed during early embryogenesis and many target genes possess DNA replication element factor (DREF)-binding sites in their promoters. In addition, we show that MSL-1 distribution remains stable across development and that binding of MSL-1 on X-chromosomal genes does not correlate with transcription in male salivary glands. These results show that transcription per se on the $\mathrm{X}$ chromosome cannot be the sole signal for MSL-1 recruitment. Furthermore, genome-wide analysis of the dosage-compensated status of $\mathrm{X}$-linked genes in male and female shows that most of the $\mathrm{X}$ chromosome remains compensated without direct MSL-1 binding near the gene. Our results, therefore, provide a comprehensive overview of MSL-1 binding and dosage-compensated status of X-linked genes and suggest a more global effect of MSL complex on X-chromosome regulation.

[Keywords: MSL; X chromosome; chromatin; dosage compensation; transcription]

Supplemental material is available at http://www.genesdev.org.

Received December 22, 2005; revised version accepted February 7, 2006.

Dosage compensation ensures that, despite unequal numbers of $\mathrm{X}$ chromosomes, expression levels of $\mathrm{X}$-linked genes are equal in males and females. During evolution, this process has evolved in different ways. In mammals, it is achieved by inactivation of one of the two female X chromosomes. In Caenorhabditis elegans, the two X chromosomes in hermaphrodites are repressed by a half. In contrast, in Drosophila, dosage compensation occurs by increasing transcription of most of the genes on the single male $\mathrm{X}$ chromosome (for review, see Baker et al. 1994). Genetics studies have led to the characterization of five genes, male specific lethal-1, male specific lethal-2, male specific lethal-3, male absent on the first (mof), and maleless (mle), all required for male viability. The products of these genes, as well as two noncoding RNAs (roX1 and roX2) assemble in a large complex, the dosage compensation complex (DCC), or MSL complex, which specifically targets hundreds of

\footnotetext{
${ }^{4}$ Corresponding author.

E-MAIL akhtar@embl.de; FAX 06221-387-518.

Article published online ahead of print. Article and publication date are at http://www.genesdev.org/cgi/doi/10.1101/gad.377506.
}

sites on the male X chromosome (Lucchesi et al. 2005; Straub et al. 2005a; Taipale and Akhtar 2005), together with the JIL1 protein, shown to be associated with the DCC (Jin et al. 2000).

Interestingly, this hyperactivation of the X-linked genes in males seems to be at least partially achieved at the level of chromatin. This is highlighted by the fact that at least two of the six proteins known to be involved in dosage compensation possess the ability to modify histones: MOF acetylates Lys 16 of histone H4 (Hilfiker et al. 1997; Akhtar and Becker 2000), while JIL1 phosophorylates Ser 10 of histone H3 (Wang et al. 2001). Both modifications have been previously linked to the regulation of transcription and chromatin structure (Turner 1998; Nowak and Corces 2004) and are significantly enriched on the male $\mathrm{X}$ chromosome in Drosophila (Bone et al. 1994; Jin et al. 2000).

One of the most intriguing aspects of dosage compensation is how this complex is able to target specifically hundreds of sites primarily on the $\mathrm{X}$ chromosome in order to ensure dosage compensation. Immunofluorescence studies on polytene chromosomes isolated from 
the salivary glands of male larvae have been used to study the targeting of the DCC on the X chromosome. According to these studies, the MSLs are distributed on hundreds of sites along the $\mathrm{X}$ chromosome, separated by gaps deprived of MSLs.

It has been proposed that the association of the MSL proteins on the $\mathrm{X}$ chromosome can be subdivided into two basic steps. The first step would include male-specific targeting of the MSL members to discrete sites on the chromosome (the "chromatin entry sites"), while the second step would involve the subsequent spreading of the complex, in a bidirectional but noncontinuous manner, to the flanking chromatin. This proposal came, first, from the observation that $\sim 35-40$ sites along the $\mathrm{X}$ chromosome can still retain MSL-1/MSL-2 in msl3, mle, and mof mutant backgrounds. Only two of these sites are fully characterized, and they correspond to the roX1 and roX2 genes. Importantly, they are able to attract the MSL complex when inserted on an autosome, leading to the theory of the "chromatin entry sites" (Lyman et al. 1997; Kelley et al. 1999). Second, the MSL complex, when targeted on an autosome by the insertion of a roX1 transgene, has the ability to spread over the surrounding chromatin (Kelley et al. 1999). However, this model appears now to be too simple. Indeed, any piece of the $\mathrm{X}$ chromosome is able to attract the complex when moved onto an autosome, even without any of the 35-40 "entry sites" (Oh et al. 2003; Fagegaltier and Baker 2004). Another model to explain MSL targeting has emerged from a recent study, which showed that recruitment of the MSLs can be induced by transcription activation of a transgene inserted in a band normally deprived in MSL (Sass et al. 2003). In addition, the distribution of the MSL complex on the $\mathrm{X}$ in ovaries is distinct from the distribution reported for salivary gland polytene squashes (Sass et al. 2003), indicating that the DCC distribution could reflect cell type expression patterns. These data suggested that transcription activation on the $\mathrm{X}$ chromosome by itself could act as a signal to recruit the DCC.

Another intriguing and poorly understood aspect of this process is how the twofold up-regulation of X-linked genes is achieved. MSL recruitment appears to be causal for transcriptional activation. Indeed, MOF is able to relieve chromatin-mediated repression of transcription in vitro and in vivo when targeted to a promoter (Akhtar and Becker 2000). Moreover, targeting the DCC artificially on an autosome leads to up-regulation of the downstream gene (Henry et al. 2001; Park et al. 2002). However, the transcriptional activation performed by the MSL complex should not overcome individual gene regulation, linked to developmental pathways, but rather fine-tune the transcription to ensure dosage compensation. How this twofold fine-tuning of transcription is achieved remains unclear. More specifically, whether the recruitment of the MSLs on X-linked genes at a local level is both necessary and sufficient to ensure a twofold overactivation of the target gene is still unknown. Apart from roX genes, only a few other X-linked genes have been shown to be targeted by the MSL complex. Chromatin immunoprecipitation (ChIP) studies have shown that the DCC is enriched on the $z w$ gene, known to be dosage-compensated, and is not present on the runt gene, compensated by an MSL-independent process (Smith et al. 2001). However, some genes, such as $1 s p 1 \alpha$, have been reported to not be compensated at all, although they are located in bands highly enriched in DCC as assessed by immunofluorescence. Conversely, the sgs 4 gene, compensated in a DCC-dependent manner (Chiang and Kurnit 2003), is located on the 3C10 cytological band, which is depleted in MSL proteins (Demakova et al. 2003).

ChIP followed by microarray (ChIP-chip) has been performed in a diverse array of taxa and has provided a comprehensive view of the distribution of a variety of transcription factors, coactivators, corepressors, or histone modifications (van Steensel 2005). These genome-wide analyses have been shown to be particularly powerful in correlating the structure of chromatin with its functional state, and in understanding modes of targeting of different factors. Given the exciting potential of these techniques with respect to the targeting of the MSL complex and the dosage compensation mechanism, we decided to utilize it to analyze the distribution of MSL-1 along the $\mathrm{X}$ chromosome, and to compare this with expression levels in males and females. To test whether MSL-1 distribution reflects cell type or developmental expression pattern, we have mapped the distribution of MSL-1 in overnight embryos, as well as in 4-6-h early embryos and in salivary glands from larvae. We also analyzed expression profiles in salivary glands from male and female third instar larvae using microarrays, to study the correlation between the presence of the complex, the transcriptional state, and the "dosage-compensated" state of X-linked genes.

\section{Results}

\section{Mapping of the DCC distribution in embryos}

In order to map the in vivo target genes of the DCC, we first performed ChIP-chip analysis from 0-14-h wildtype embryos with specific antibodies against MSL-1. The DNA amplified from the MSL-1 immunoprecipitation was labeled with Cy5 fluorophore and hybridized against DNA amplified from a nonspecific immunoprecipitation (mock IP), labeled with Cy3 dye. We used a cDNA array covering the DGC1 and DGC2 cDNA libraries from the Berkeley Drosophila Genome Project (see Materials and Methods), which represents $>70 \%$ of the coding sequences of the Drosophila genome (Stapleton et al. 2002). Of the 10,188 spotted clones that have chromosomal annotation, 1699 (i.e., 16.6\%) are located on the X chromosome, representing $73.6 \%$ of the annotated features on the $\mathrm{X}$ (based on Flybase version 4.2.1 annotation). All raw data are publicly available on Array Express (http://www.ebi.ac.uk/arrayexpress/query/entry, accession no. E-MEXP-554). For comparative analysis MSL-3 IP was performed in parallel (Supplementary Table 1).

The roX1 gene has previously shown to be enriched for the MSL proteins in many systems (embryos, third instar larvae salivary glands, SL-2 cells) (Smith et al. 2001) and, 
therefore, can serve as an internal positive control for the arrays. Consistent with previous observations we also found that roX1 was significantly enriched for both MSL-1 and MSL-3 ( $p=0.00025$ for MSL-1, $p=0.01556$ for MSL-3) in our arrays. The runt gene, previously reported to be depleted of MSL complex, was used as a negative internal control and was not significant for either MSL-1 $(p=0.68)$ or MSL-3 $(p=0.82)$. In addition, we performed quantitative PCR (qPCR) on 20 clones for further validation. There was a significant correlation between the enrichment ratios (MSL-1/mock) obtained by qPCR and the microarray $\left(r=0.77, p\right.$-value $\left.<10^{-4}\right)$ (Supplementary Fig. 1).

To identify the candidate MSL-1 target population, we used the joint criteria of statistical significance and rank ( $p$-value $<0.1$ and median percentile rank across the arrays $>95 \%$ ) (Supplementary Table 2). After removal of the clones that do not have chromosomal annotation, $82 \%$ of the clones identified by these joint criteria 196 out of 117) were located on the X chromosome, in agreement with immunofluorescence studies on polytenes chromosomes (Fig. 1A). It is interesting to note that the MSL complex has been reported to interact also with a few sites on autosomes (Demakova et al. 2003), which could explain the presence of autosomal genes in the MSL-1 target clone subset.

A sample $(n=7)$ of clones from the candidate target list on the $\mathrm{X}$ was selected for further validation. There was $100 \%$ confirmation of MSL-1 enrichment by qPCR (Supplementary Fig. 1). In addition, four clones located on the X chromosome that did not meet the candidate target criteria were also examined by qPCR. No significant enrichment over the mock IP was seen for these clones (Supplementary Fig. 1).

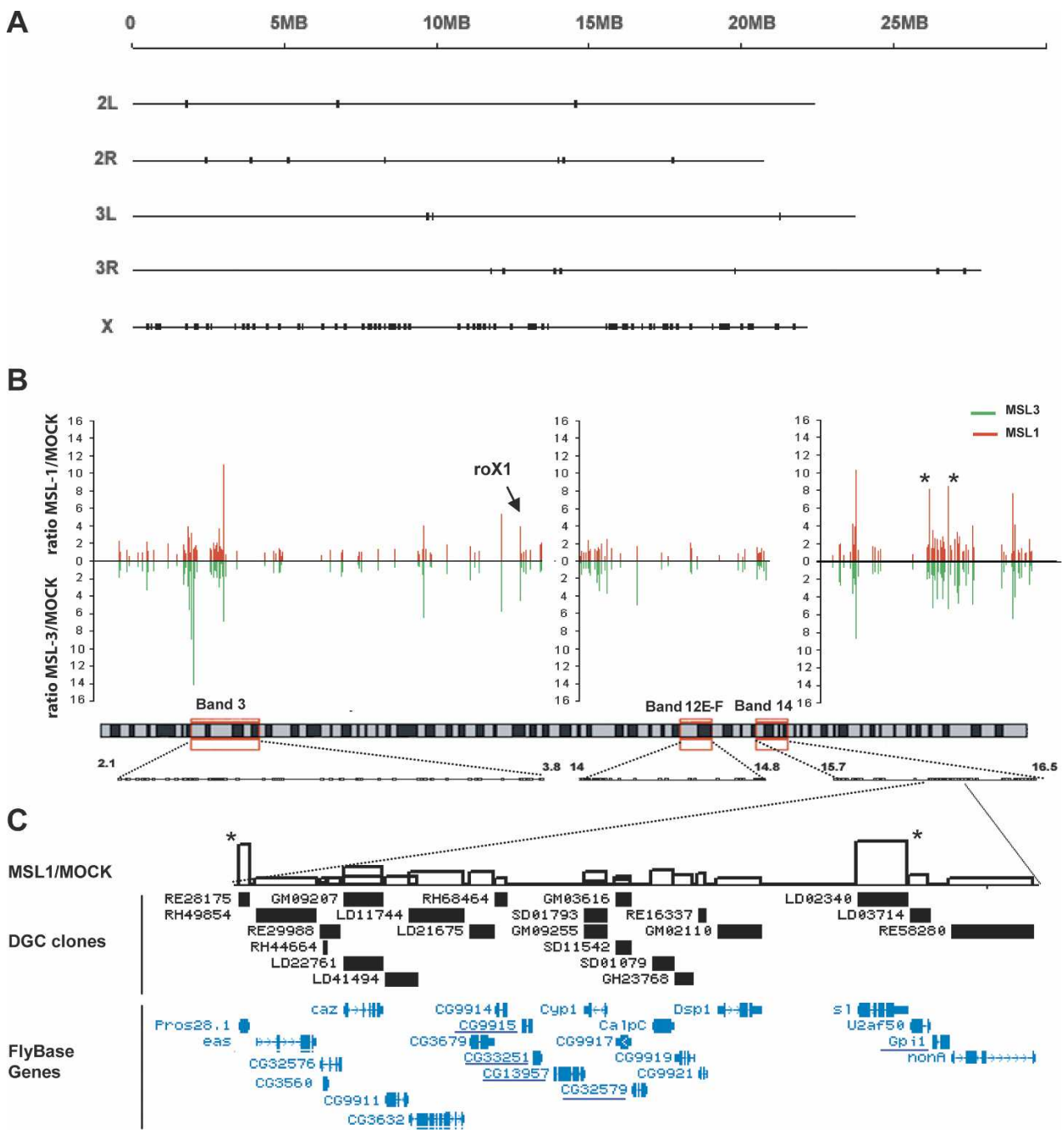

Figure 1. Binding of MSL-1 and MSL-3 in 0-14-h Drosophila embryos. (A) Chromosomal distribution of MSL-1 target genes in 0-14-h embryos, defined as the genes showing a rank percentile $>95 \%$, and a $p$-value $<0.1$. The majority of these genes are located on the $\mathrm{X}$ chromosome as shown. (B) Distribution of MSL-1 (in red) and MSL-3 (in green) on three cytological bands of the X chromosome. Each vertical line represents the average MSL-1/MOCK ratio of a probed gene at its chromosomal position (in megabases). Positions of the DGC clones spotted on the array are presented below each graph. The roX1 gene is indicated by an arrow. Asterisk indicates cluster of genes represented in C. $(C)$ Detailed view of a cluster of genes located in the 14 cytological band. 
The distribution of MSL- 1 and MSL- 3 binding across the $\mathrm{X}$ was examined (using gene positions from the Berkeley Drosophila Genome Project release 4.1) (Fig. 1B). As expected, the distribution of MSL-1 (represented in Fig. 1B, in red) overlapped with that of MSL-3 (represented in green) (with 34 [or 42\%] of the MSL-3 targets seen in MSL-1). There was a strong correlation between the binding of MSL-1 and of MSL-3 (values expressed as $\log$ ratios) on the $\mathrm{X}\left(r=0.81, p<10^{-4}\right)$ (Supplementary Fig. 2). Finally, X-linked genes identified by our criteria as candidate targets with MSL- 1 had a median percentile rank of $94 \%$ for MSL-3, indicative of a high concordance between the target gene populations for MSL-1 and MSL-3 (Supplementary Table 2). Taken together, these results indicate that MSL-1 and MSL-3 bind highly overlapping target sites across the $\mathrm{X}$ chromosome in embryos.

Interestingly, MSL- 1 and MSL-3 appear to be targeted at the individual gene level, reflected in the varied level of MSL complex enrichment within clusters of genes (e.g., the clusters of genes on cytological bands 3 and 14 in Fig. 1B). It is of course possible that MSL- $1 / 3$ may also bind to intergenic or intronic regions, or to additional genes missing from our arrays. However, a close-up of 95 $\mathrm{kb}$ of a gene dense region well tiled on our array (18 out of the 23 genes represented, covering $\sim 66 \mathrm{~kb}$ ) confirmed this finding (Fig. 1C). For example we observed enrichment of MSL-1 on the s1 gene, whereas U2af50 shows a background enrichment level, although located $<0.5 \mathrm{~kb}$ from sl. This wide variability of MSL-1 targeting among neighboring loci shows for the first time that the complex targets discrete loci and not broad chromosome domains. In addition, these results indicate that our ChIP data provide sufficient resolution to allow further analysis of the target gene population.

Functional features of MSL-1 target genes: MSL-1 binds genes with shared expression profile and transcription factor motifs

In order to better understand the modes of MSL targeting, we decided to investigate the different functional characteristics of these X-linked genes highly enriched in MSL-1. For comparative purposes, we defined a category of X-linked "nontarget" genes ( $p$-value $>0.6$ and percentile rank $<60 \%$ for both MSL- 1 and MSL-3 data). First, considering that without dosage compensation males die, we wondered whether these target genes are essential for life. For this analysis we used the data generated by the large-scale P-element insertion screens (Bourbon et al. 2002; Peter et al. 2002). There was no significant association between the phenotype (lethal or not) and the target status (data not shown), even though we could observe a slight preference of MSL-1 targeting on essential genes, as $12.5 \%$ of the MSL- 1 targeted genes were essential whereas this was the case for only $7 \%$ of the nontargeted genes.

We next addressed whether targeting of the MSL-1 happens on genes sharing common transcription factorbinding sites. For this purpose we used the REDUCE algorithm (http://bussemaker.bio.columbia.edu:8080/ reduce) that has been designed for the discovery of regulatory elements based on microarray expression data, in an unbiased manner (Bussemaker et al. 2001). We performed REDUCE analysis on the 1-kb upstream sequences of the entire set of probed loci to discover motifs that correlate with the chromatin profiling data for MSL-1. We found an overrepresentation of motifs that are part of the DNA replication-related element (DRE), targeted by DREF (DNA replication element factor), among the most significant motifs. We further confirmed this result by comparing the target and nontarget populations for the presence of DRE elements; $28.2 \%$ of the target gene indeed possessed at least one consensus DRE site in their 3-kb upstream sequence, versus only $15.8 \%$ for the nontarget genes (Fisher exact $p=0.0034$ ).

Finally, we asked whether the MSL-1 target genes share similar expression patterns during development. For this analysis we used the Yale Drosophila developmental transcriptional time course data set (Arbeitman et al. 2002). In addition to differential gene expression analysis, cluster analysis (average linkage using a correlation metric) was performed to aid in visualization (Cluster and Treeview software programs, http://rana. lbl.gov/EisenSoftware.htm). Strikingly, most of the MSL-1 target genes for which expression data are available exhibited differential expression levels between early embryogenesis and later developmental stages, which was not observed for the nontarget population (Fig. 2). Most of them showed higher expression level until 11-12 h after fertilization (with an average log ratio of 0.46 for the target against -0.019 for the nontarget). Even when using the most stringent post-hoc multiple comparison adjustment (Bonferroni), these differences in expression were significant between target and nontarget populations at each of the seven early embryonic time points in the Arbeitman data set (up to $25 \mathrm{~h}$, all adjusted $p$-values $<0.05$ ).

Taken together, these data show that, in embryos, MSL-1 is targeted on genes sharing common functional features such as common DREF-binding sites and similar expression patterns during development.

\section{The distribution of the MSL-1 is stable between early embryos and salivary glands of third instar larvae}

The above results also suggest that targeting of MSL-1 may correlate with transcription activation, as we observe MSL-1 binding in 0-14-h embryos, on genes that are highly transcribed during early embryogenesis. Recent data have indeed led to the proposal that the MSLbinding pattern on the $\mathrm{X}$ chromosome could reflect the cell-type-specific gene expression patterns (Sass et al. 2003). In order to investigate this point in detail we decided to study whether the localization of MSL-1 changes during development. For this purpose, we compared the distribution of MSL-1 at two different developmental stages. Chromatin was prepared from tightly staged early embryos (4-6 h), where dosage compensation is just established (Rastelli et al. 1995; Franke et al. 


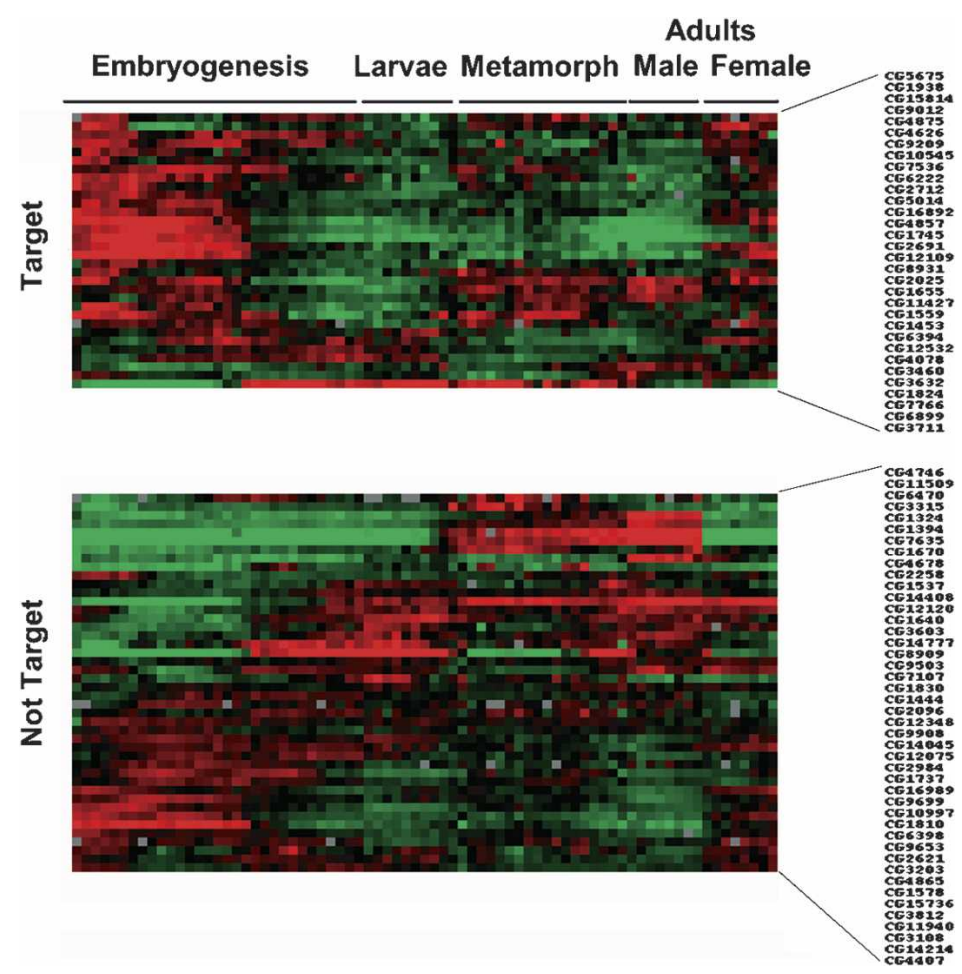

Figure 2. MSL-1 binds on active genes early in development. Clustered expression profiles of target and nontarget genes of MSL-1 in 0-14-h embryos. Most target genes are predominantly expressed in embryos. Red indicates high expression level, green indicates low expression level. Gene expression data were taken from Arbeitman et al. (2002).
1996) and from dissected male salivary glands isolated from third instar larvae. This strategy ensured chromatin preparation and further analysis on a more homogenous population. Furthermore, analyzing the distribution pattern of MSL-1 in salivary glands also allowed us to compare the results with pre-existing data of MSL-1 localization from immunofluorescence studies on polytene chromosomes.

As expected, roX1 showed a strong enrichment level in both data sets (larvae: $p=0.093$, rank $=99.2 \%$; 4-6-h embryos: $p=0.0119$, rank $=98.8 \%$ ). We used the same criteria as previously to define the subset of target genes $(p<0.1$, rank percentile $>95 \%)$. For salivary glands and 4-6-h embryos, $86 \%$ and $89 \%$ of the clones following these criteria were located on the $\mathrm{X}$ chromosome, respectively (i.e., 197/229 and 407/457 that possess chromosomal annotation, respectively; Supplementary Table 2). The cytological region 12E6-12F3 previously reported to be depleted of MSL proteins on polytene squashes (Demakova et al. 2003) appeared depleted of MSL-1 also in our salivary glands ChIP-chip analysis, further validating the results (Fig. 3A).

Interestingly, MSL-1 targeting in 4-6-h embryos is correlated with MSL-1 targeting in larvae on the X chromosome $\left(r=0.69, p<10^{-4}\right)$ (Fig. 3B). Among the 407 clones target in 4-6-h embryos, $40 \%$ (i.e., 163) were still able to attract the complex in larvae, representing $82.7 \%$ of the larvae target population. This strong overlap seen between the two populations of target genes indicated that most of the genes targeted by MSL-1 in larvae appear to have already been targets in 4-6-h embryos.

Taken together, these results indicate that the pattern of MSL-1 binding is set up early in development, with
MSL-1 binding in later stages presenting a subset of binding in earlier stages. This might also explain why we could identify many more MSL-1-binding sites in 4-6-h embryos compared with overnight embryos and larvae, although this difference could also be related to different efficiencies of immunoprecipitation.

\section{Correlation between MSL-1 targeting and transcription}

The above results argue against a correlation between transcription and targeting since many genes expressed during embryogenesis undergo inactivation during larval stages (Arbeitman et al. 2002), whereas MSL-1 appears stably bound on the $\mathrm{X}$ chromosome across development. We, therefore, further investigated the relationship between transcription activation and MSL-1 targeting.

First, we compared our ChIP-chip data with the expression data available on the Yale Drosophila lifetime course (Arbeitman et al. 2002). The scatter plots presented in Figure 4A show the correlation between targeting (log ratio MSL-1/MOCK) and the transcription (log ratio time point/pool) for all the DGC clones on the $\mathrm{X}$ chromosome. We found a weak correlation between transcription and MSL-1 binding in 4-6-h embryos (ranging from $r=0.29-0.33$ depending on the time point considered [4-5 h or 5-6 h], $p<10^{-4}$ ), but surprisingly we did not find any biologically significant correlation when we compared our ChIP-chip results in third instar larvae salivary glands with the 96 -h or 105 -h expression time points (respectively, $r=-0.09, p=0.04 ; r=-0.04, p=0.3$ ) (Fig. 4A, left and middle panels). However, the differences could be due to the fact that previous expression profiles were generated using whole larvae (Arbeitman et 
Legube et al.

Figure 3. Similar distribution of MSL-1 binding between 4-6-h embryos and salivary glands of third instar larvae. (A) Distribution of MSL-1 in 4-6-h-staged embryos (in red) and in male third instar larvae salivary glands (in blue) on the same three cytological bands of the $\mathrm{X}$ chromosome represented in Figure 1B. $(B)$ Bivariate scatterplot of MSL-1/MOCK log ratio in 4-6-h embryos and in third instar larvae salivary glands of all probed loci on the $\mathrm{X}$ chromosome.

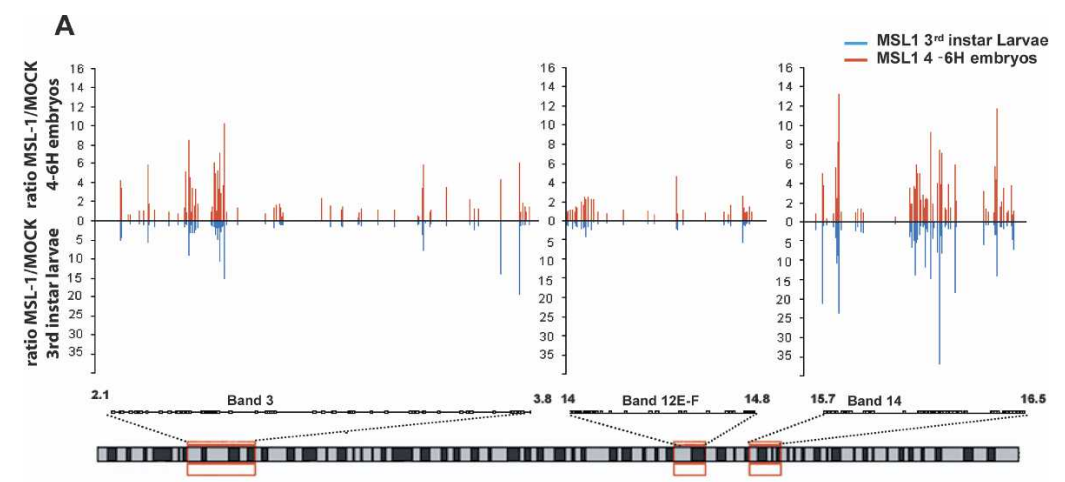

B

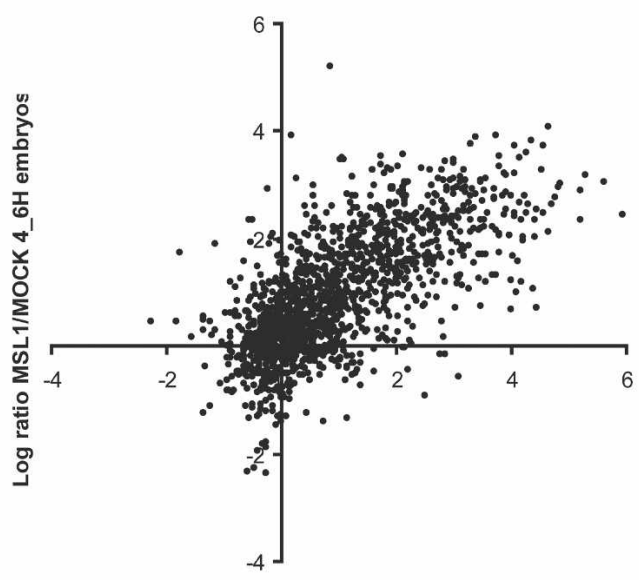

Log ratio MSL1/MOCK larvae

ously selected, 884 showed an RNA level in salivary glands above background threshold levels (based on negative controls) defining our class of expressed genes, whereas $151 \mathrm{did}$ not, thus representing a not expressed class of genes. The MSL-1 targeting status of genes in both sets (expressed and nonexpressed) was examined. We observed a significant association between targeting and transcription (Fisher's exact $p=0.023$ ) with a preference for the target genes to be transcribed. However, 13 out of the 149 MSL-1 target clones were not expressed (i.e., $8.7 \%$ ), indicating that targeting of the MSL-1 by itself does not induce transcription activation, at least not to a level we could detect in our experiments.

It was surprising that only 136 of the 884 expressed genes (i.e., 15.4\%) were bound by MSL-1, indicating that expression of $\mathrm{X}$-linked genes does often not lead to MSL-1 binding. Since we cannot rule out that we may have missed some target sites due to the stringency of our criteria as well as the nature of the cDNA arrays, it remains to be seen what proportion of the remaining expressed genes may be targeted by MSL-1.

We also performed immunostaining of polytene chromosomes isolated from salivary glands of third instar larvae, using MSL-1 antibody in combination with Spt5, Spt6 (elongation factors), or PolII-S5-P antibody (active form of the RNA polymerase II). Strikingly, this analysis, even though allowing a lower resolution than the ChIPchip, also showed a distinct binding pattern of MSL-1 
A

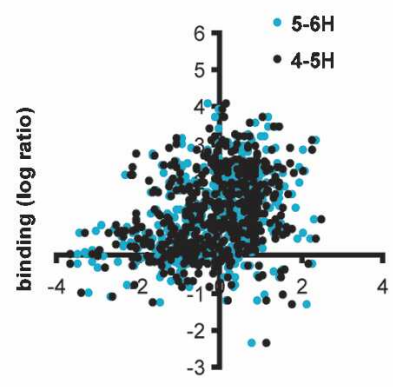

expression (log ratio)

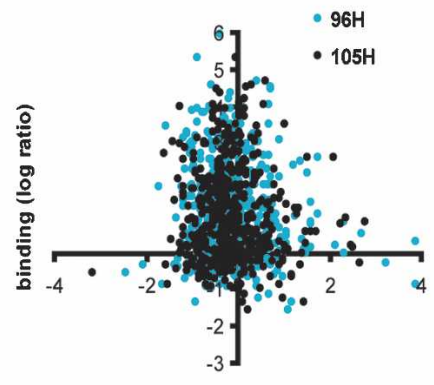

expression (log ratio)

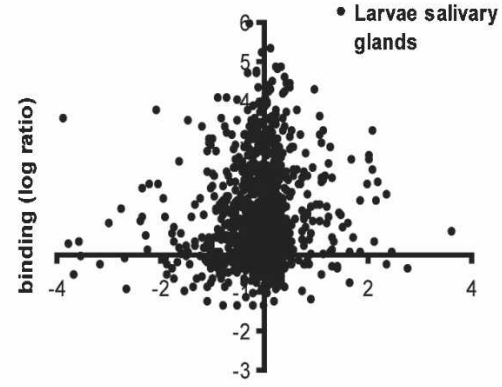

expression (log ratio)

B
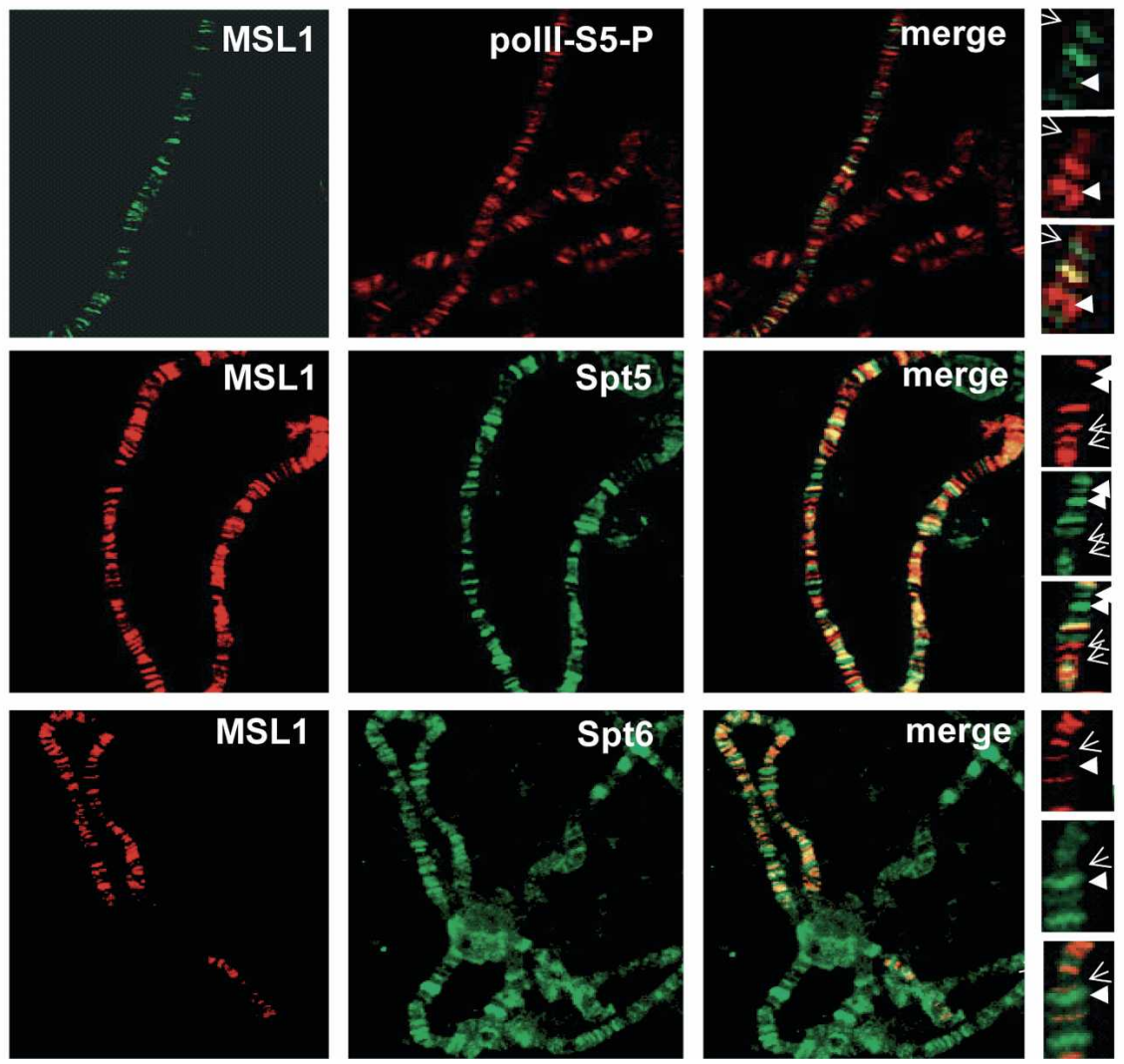

Figure 4. MSL-1 binding correlates poorly with transcription in male salivary glands. (A) Bivariate scatterplots of MSL-1/MOCK ChIP data and time point/pool expression data. MSL-1/MOCK log ratio is obtained from the 4-6-h ChIP-chip data set (left panel) and from the male third instar salivary glands ChIP-chip data set (middle and right panels). Time point/pool log ratio are obtained from the 4-5-h (in black) and 5-6-h (in blue) time points (left panel); from the 96-h (in blue) and 105-h (in black) time points (middle panel) (Arbeitman et al. 2002); and from the male salivary gland expression data set (analyzed from four independent experiments, our results) (right panel). (B) Immunofluorescence analysis of the distribution of MSL-1 and of three transcription-associated factors on male polytene chromosomes from third instar larvae Spt5, Spt6, and RNA polymerase II (PolII-S5-P; phosphorylated on the Ser 5 of the CTD).

and other transcription-associated factors in salivary glands. Spt6, Spt5, or PolII-S5-P could immunostain bands depleted in MSL-1, and vice versa (Fig. 4B), further supporting the array data.

Taken together, these results show that even though MSL-1 preferentially binds active genes, transcription per se is not sufficient or required for MSL-1 localization on the $\mathrm{X}$ chromosome.
Correlation between dosage compensation and MSL-1 binding

Next we were interested to study the dosage compensation status of the X-linked genes that are bound or not bound by MSL-1, since we found high proportion of transcribed genes not bound by MSL-1. For this purpose we purified RNA from female salivary glands and hybridized 
it, against the same pool of RNA as previously used for male expression profile analysis.

The expression data obtained for female are highly similar to that seen in male (Fig. 5A) $\left(r=0.94, p<10^{-4}\right)$, suggesting that most genes are compensated on the $\mathrm{X}$ chromosome, at least in salivary glands of third instar larvae. Since studying twofold expression level differences is difficult in an array experiment, we imposed stringent criteria to define a "dosage-compensated" subset (FDR adjust $p=1$ ) to ensure further analysis on a more strict population, even if it was at the expense of missing a fraction of dosage-compensated genes. Using these criteria we found that 588 out of the 884 transcribed genes are compensated, representing $\sim 66.4 \%$ of the transcribed genes.

When we compared target and dosage compensation status we found that there was no difference in the distributions, with $72 \%$ of the target and $68 \%$ of the nontarget populations considered to be dosage-compensated. Even though there is a slight tendency for the target population to be dosage-compensated, these results indicate that there is no clear relationship between dosage-compensation and MSL-1 target status in salivary glands.

Since we were concerned that, even after using restrictive criteria based on FDR-adjusted $p$-values, we may still overrepresent the dosage-compensated population, we performed qPCR on a set of X-linked genes, showing different MSL-1 enrichment status. Among the 16 genes we tested, all the genes showing an FDR adjust $p=1$ in our array experiment (Fig. 5B, in blue) also appeared compensated by qPCR analysis, validating the criteria we applied previously to define our dosage-compensated subset. In addition, genes that showed a low FDR $p$-value $(p<0.1)$ together with female-biased expression (in red) also appeared to be not compensated by qPCR. BR-C represents an example of the genes that we missed by applying stringent criteria (FDR $p=1)$ to define the dosage-compensated population. Indeed, although this gene has been excluded from our dosage-compensated subset

A

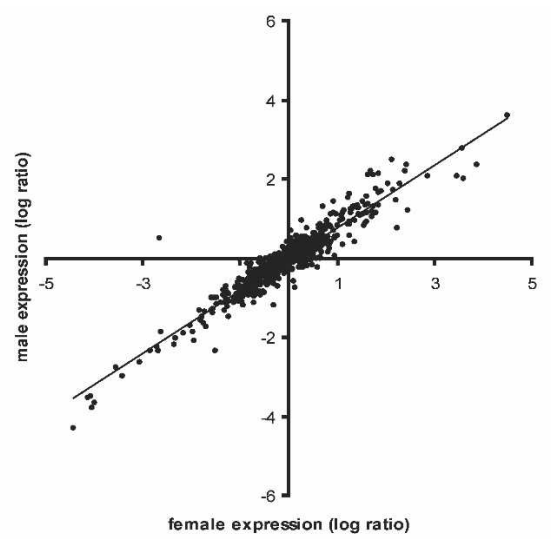

B

Figure 5. Direct MSL-1 binding is uncoupled at a local level with dosage compensation. $(A)$ Bivariate scatter plot of the expression data (expressed as log ratio) obtained from male and female salivary glands, for the clones located on the X chromosome. (B, top) qPCR analysis of the RNA level in third instar salivary glands, in male and female, of nine genes "targeted" by MSL-1 according to our ChIP-chip analysis in larvae $(p<0.1$, rank percentile $>95 \%)$, and seven genes defined as "not targeted" in salivary glands $(p>0.6$, rank percentile $<60 \%)$. The genes that were part of the dosage-compensated subset according to the expression array data are shown in blue (FDR $p=1)$, whereas the genes in red showed an adjusted FDR $p$ value $<0.1$; i.e., female-biased expression. All RNA levels were normalized against GAPDH. Male RNA levels are expressed as percentage of female expression level set at 100\%. (Bottom) Binding profile of MSL-1 on the corresponding genes in the top panel represented by fold enrichment of MSL-1/MOCK in ChIPchip salivary glands data set.

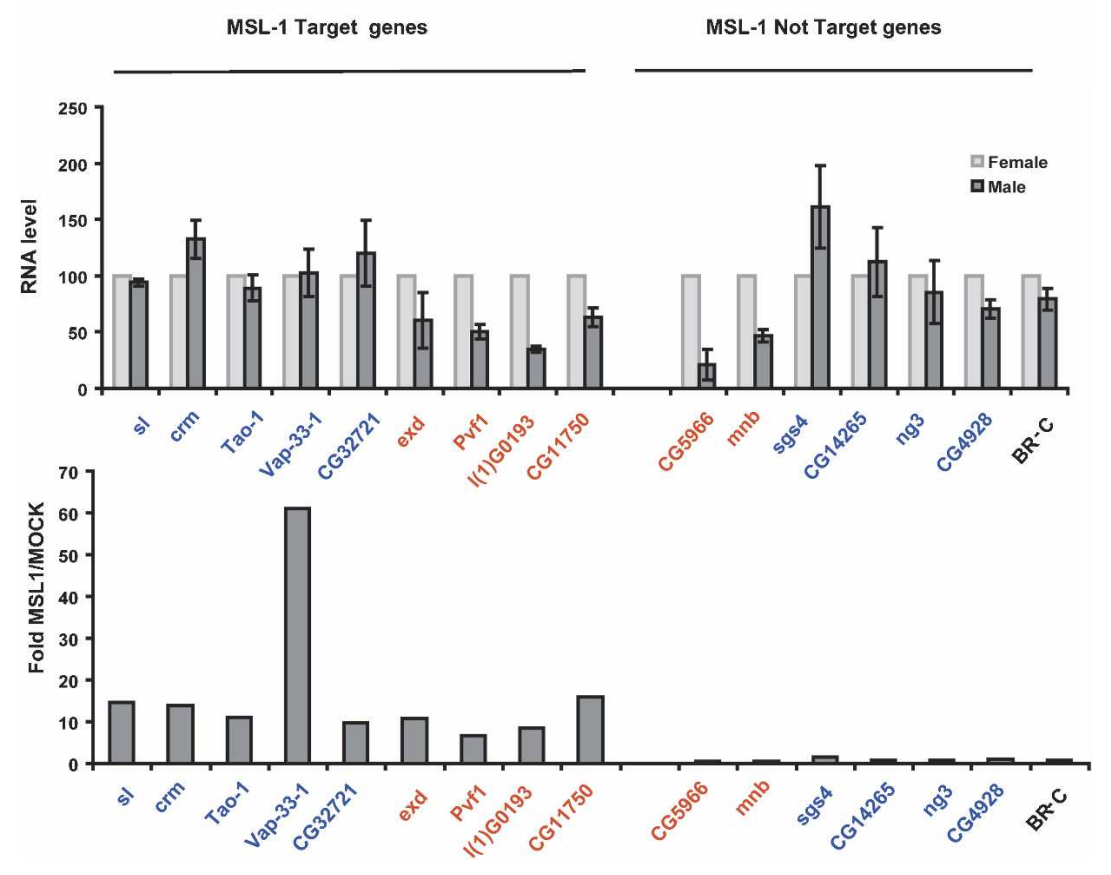


since it showed an FDR $p=0.96$, it appeared compensated by qPCR analysis.

As observed using our microarray data, the enrichment status did not always correlate with the dosagecompensated state. Among the nine genes of the target subset that we tested in qPCR, five (sl, crm, tao-1, vap33-1, and CG32721) were either expressed at equal level in male and female (i.e., dosage-compensated), or slightly higher in male than in female. However, four genes $(e x d$, pvf1, 1(1)G0193, and CG11750) did not appear to be dosage-compensated since they showed twofold lower expression in male than in female (Fig. 5B, genes "target"), indicating that some genes can escape dosage compensation, even though they are bound by the MSL complex.

Among the seven genes scored as MSL-1 "not target" $(p>0.6$ and rank percentile $<60 \%)$ that we tested by qPCR, 2 (mnb and CG5966) showed lower expression in males compared with females. However, five genes (sgs4, CG14265, ng3, CG4928, BR-C) showed similar expression between male and female (i.e., dosage-compensated), or a tendency to be higher expressed in male. Taken together, these data show that both MSL-1-bound and unbound populations are comprised of dosage-compensated and nondosage-compensated genes.

\section{Discussion}

In this study we report genome-wide profile of MSL-1 distribution along the $\mathrm{X}$ chromosome in embryos and salivary glands isolated from third instar larvae. Our results show that most MSL-1-bound genes are expressed early in embryogenesis. The MSL-1 distribution appears to be set up early in development. Intriguingly, we find that even though most of the MSL- 1 target genes are transcribed, target genes account for only a subset of transcribed genes on the $\mathrm{X}$ chromosome. This indicates that binding of MSL-1 on an X-linked gene is not a prerequisite for transcription activation in larvae, and that transcription on the $\mathrm{X}$ by itself cannot be the sole signal to attract the DCC. Furthermore, we found that a high proportion of both MSL-1 target and nontarget genes appears dosage-compensated, suggesting a global role of the MSL complex in X-chromosomal regulation.

\section{Targeting of the MSL complex on the X chromosome}

Using the ChIP-chip strategy, we examined the MSL-1 distribution on the Drosophila genome in order to investigate how the MSL complex achieves specific targeting on the $\mathrm{X}$ chromosome. Our analysis provides a first comprehensive list of MSL-1-binding sites along the X chromosome in early embryos and male salivary glands. Furthermore, we show that MSL-1 binds individual gene loci rather than broad chromosomal domains, which could not be addressed previously (Straub et al. 2005b).

More specifically, we wanted to address whether or not binding of MSL-1 is directed by transcription activation, since it was recently shown that driving transcription from a transgene inserted on the $\mathrm{X}$ chromosome is able to induce local DCC recruitment (Sass et al. 2003). Our results show that even though the MSL complex is predominantly bound on transcriptionally active genes, transcription of endogenous X-linked genes per se is not sufficient to attract the complex in salivary glands. This conclusion is based on the following observations. The first evidence is provided by the fact that the MSL-1 distribution is highly stable between two tissues as different as whole 4-6-h embryos and third instar salivary glands, indicating that the MSL distribution is unlikely to reflect expression profiles. Indeed, $40 \%$ of the target genes in 4-6-h embryos were still bound by MSL-1 in larvae, which represent most (82.7\%) of the MSL-1 target genes in salivary glands. Furthermore by comparing expression and MSL-1-binding profiles in male salivary glands, we observe that recruitment of the MSL complex is not a general property of active X-linked genes, since only $15 \%$ of transcribed genes present on our array are directly bound by MSL-1. It remains possible that we may have missed a number of target sites in the intergenic or intronic regions since our analysis was performed on cDNA arrays. However, the comparison of the MSL-1-binding pattern with transcription-associated factors such as Spt-5, Spt-6, and S5-P PolII on intact polytene chromosomes also did not reveal a strong overlap between these proteins and MSL-1. These results are also consistent with recent observations (Kotlikova et al. 2006) that these transcription-associated factors localize both in bands enriched in MSL-1 and in bands depleted of MSL-1.

How is then the MSL complex targeted on the X chromosome? It is possible that transcription on the $\mathrm{X}$ acts as a signal for MSL-1 targeting during early stages of development. Once bound, the complex would then either be stabilized on the target genes or decay, independently of further changes in expression (accounting for the absence of major developmental change in the DCC distribution). In favor for this model we observed that MSL-1 binding correlates more with transcription in early embryos $(r=0.3)$ than in larvae $(r=0.1)$ (Fig. 4A), and that most of the MSL-1 target genes in all the stages we tested show high expression levels during early embryogenesis (Fig. 2; data not shown).

Alternatively, MSL-1 could be targeted by specific transcription factors to a small subset of genes. Indeed we observed that MSL-1 target genes are enriched in DREF-binding sites. DREF had previously been identified as a potential regulator of genes involved in cell cycle and growth regulation. DREF associates in vivo with the core promoter transcription complex TRF2 (TBP-related factor 2) and it has been proposed that it may target TRF2 to a subset of core promoters (Hochheimer et al. 2002). In a similar way DREF may promote gene selectivity for the DCC and act as a DNA-targeting component for the DCC. Consistent with this hypothesis, we found that the $\mathrm{X}$ chromosome is enriched in comparison to autosomes in genes that possess several DRE or DRE-related sites in their 2-kb upstream sequence (data not shown). Another interesting candidate would be the GAGA factor (GAF), which has been shown 
to colocalize to some extent with the MSL complex on polytene chromosomes, and which is required for the proper localization of MSL on the $\mathrm{X}$ chromosome (Greenberg et al. 2004). It is noteworthy that the transgenes that were previously shown to attract the MSL complex when transcribed also possessed a few GAFbinding sites (Sass et al. 2003). A careful analysis of our MSL-1 distribution in 0-14-h embryos revealed that MSL-1 target genes are slightly more enriched in GAFbinding sites than nontarget genes (G. Legube, S.K. McWeeney, and A. Akhtar, unpubl.).

Targeting of MSL proteins on selected X-linked genes could then be achieved by a combinatorial effect of several independent motifs. This may explain why previous attempts to find specific X-chromosomal consensus sequences were not successful. Therefore, ChIP-chip approaches such as used in this study, which allow the discovery of physiological targets in an unbiased and global manner, will be important to unravel the complexity hidden within this system.

\section{Role of the MSL complex in transcription activation and dosage compensation}

In addition to providing insights into the MSL-1 targeting mechanism, our data also raise important questions about the role of the MSL complex in transcription activation and in the dosage compensation mechanism. First of all, according to our data, $>66 \%$ of the transcribed X-linked genes are compensated, whereas only $15 \%$ are bound by MSL-1. Moreover, our array and qPCR analyses suggest that local MSL-1 binding is neither sufficient nor necessary to ensure dosage compensation in salivary glands.

One possibility is that the genes not bound by the MSL complex, but still compensated, could be up-regulated by an MSL-independent mechanism. However, we consider this possibility unlikely, as such an MSL-independent mechanism would have been already discovered by genetic screens (since it would then concern most of the dosage-compensated genes). In addition most of the genes on the $\mathrm{X}$ chromosome seem to be compensated in an MSL-2-dependent manner (Hamada et al. 2005). Therefore, we favor the idea that the MSL complex could have long-range effects on the transcription of X-linked genes. It has been already reported that the $S g s 4$ and $B R-C$ genes are dosage-compensated in third instar larvae in an MSL-dependent manner (Chiang and Kurnit 2003). Both of them are not bound by MSL-1 according to our data, and moreover Sgs 4 is localized in a band depleted in MSLs as assessed by immunofluorescence on polytene chromosomes (Demakova et al. 2003), supporting the idea of an action of MSL in a long-range manner.

How the MSL complex could operate to fine-tune the transcription of genes, distant from the direct MSL-binding sites, still remains a mystery. One exciting possibility may be that the MSL complex is actually required to recruit parts of the $\mathrm{X}$ chromosome into a nuclear domain with unique transcriptional/post-transcriptional proper- ties. It has become clear that spatial positioning within the nucleus also plays a central role in the control of gene expression, allowing the coregulation of subsets of genes (Schubeler et al. 2000; Ragoczy et al. 2003; Chambeyron and Bickmore 2004). MSL binding on discrete loci could induce the localization of a broad X-chromosomal domain, containing several genes, to a nuclear compartment that possesses specific transcriptional properties. This may ensure the dosage compensation of many Xlinked genes, without a need of direct MSL binding on these genes. Interestingly, purification of the MSL complex has revealed coassociation of several nucleoporins in embryos and Schneider cells (Mendjan et al. 2006). One may envisage that concerted action of MSL with nuclear pore components may help to define such domains needed for cis-regulation of many genes.

An interesting feature of the dosage-compensated Xchromosome includes the colocalization of MSL proteins with specific histone H4 Lys 16 acetylation (H4K16Ac). This histone modification has been proposed many times to be related to transcription activation and appears to have a unique, although still poorly understood, role in transcriptional regulation (Dion et al. 2005). In Drosophila, H4-K16 acetylation overlaps with MSL binding on the male X chromosome on polytene chromosomes (Turner et al. 1992), and driving artificially H4-K16 acetylation by MOF is able to increase transcription (Akhtar and Becker 2000). However, the situation for endogenous X-linked genes appears to be more complex, at least in salivary glands, since we were able to find a number of genes bound by MSL-1 that did not seem to be transcribed (Fig. 4B). Interestingly, and in agreement with our observation in Drosophila, genomewide analysis in Saccharomyces cerevisae showed that the distribution of H4-K16 acetylation along the chromosomes does not correlate with transcriptional activity (Kurdistani et al. 2004). Therefore, the function of H4K16 acetylation in the dosage compensation process and more generally in transcriptional regulation appears unclear.

Another important issue to consider is that Drosophila salivary glands, although differentiated, undergo endoreplication (for review, see Edgar and Orr-Weaver 2001). Global analyses revealed that there is a correlation between replication timing and transcription activity. However, this correlation is not absolute (Schubeler et al. 2002; MacAlpine et al. 2004; Belyakin et al. 2005). Furthermore, similar replication timing profiles were obtained in two human cell types as different as fibroblasts and lymphoblasts, expected to show quite different gene expression profiles (White et al. 2004). Since H4 hyperacetylation has been associated with active replication origins (Aggarwal and Calvi 2004), it is tempting to speculate that MSL/H4-K16Ac distributions may correlate with replication timing. Interestingly, the DREF transcription factor, which binds the DRE sequence, identified in our MSL-1 target genes, plays a role in endoreplication in salivary glands (Hirose et al. 1999). Future global analyses of binding profiles of all MSL components together with the comparison of profiles of other 
transcription/replication factors and histone modifications will certainly help our understanding of H4-K16 acetylation and dosage compensation in Drosophila.

\section{Materials and methods}

\section{ChIP}

Chromatin was prepared either from overnight 0-14-h embryos or tightly staged 4-6-h embryos (after two rounds of 1-h prelay) according the protocol developed by Orlando et al. (1997). The fragments generated were 500 base pairs $(\mathrm{bp})$ in average length. Chromatin from salivary glands of third instar larvae was prepared using the following procedure: Salivary glands (10 per ChIP) were fixed for $15 \mathrm{~min}$ at room temperature in $1 \mathrm{~mL}$ of fixing solution $(50 \mathrm{mM}$ HEPES at $\mathrm{pH} 7.6,100 \mathrm{mM} \mathrm{NaCl}$, $0.1 \mathrm{mM}$ EDTA at $\mathrm{pH} 8,0.5 \mathrm{mM}$ EGTA at $\mathrm{pH} 8,2 \%$ formaldehyde) on a rotating wheel. The salivary glands were then centrifuged at $2000 \mathrm{rpm}$ for $1 \mathrm{~min}$, washed once in PBS supplemented in $0.01 \%$ Triton X-100 and $0.125 \mathrm{M}$ glycine, and then washed sequentially for 10 min each in $1 \mathrm{~mL}$ of $0.25 \%$ Triton $\mathrm{X}-100,10 \mathrm{mM}$ EDTA (pH 8), $0.5 \mathrm{mM}$ EGTA (pH 8), and $10 \mathrm{mM}$ Tris (pH 8), and in $200 \mathrm{mM} \mathrm{NaCl}, 10 \mathrm{mM}$ Tris $(\mathrm{pH} 8), 10 \mathrm{mM}$ EDTA (pH 8), and $0.5 \mathrm{mM}$ EGTA (pH 8). The glands were then resuspended in $500 \mu \mathrm{L}$ of sonication buffer $(10 \mathrm{mM}$ Tris at $\mathrm{pH} 8$, $1 \mathrm{mM}$ EDTA at pH 8, $0.5 \mathrm{mM}$ EGTA at $\mathrm{pH} 8.0$ ) and sonicated 8 min (pulsed eight times for $30 \mathrm{sec}$, paused for $30 \mathrm{sec}$, high) in a Bioruptor (Cosmo Bio). Samples were then adjusted in $0.5 \%$ sarcosyl, incubated on a wheel for $10 \mathrm{~min}$ at room temperature, centrifuged at 14,000 rpm for $10 \mathrm{~min}$, and dialyzed overnight at $4^{\circ} \mathrm{C}$ against $5 \%$ glycerol, $10 \mathrm{mM}$ Tris $(\mathrm{pH} 8), 1$ mM EDTA $(\mathrm{pH}$ 8 ), and $0.5 \mathrm{mM}$ EGTA (pH 8). The fragments generated were 500 bp on average.

Immunoprecipitation was then performed on $500 \mu \mathrm{L}$ of chromatin from embryos or salivary glands, according to Orlando et al. (1997), using $3 \mu \mathrm{L}$ of a polyclonal antibody against MSL-1 (kindly provided by John Lucchesi, University of Atlanta, Atlanta, GA), a polyclonal antibody against MSL-3, or a preimmune rabbit anti-serum (mock ChIP). The immunoprecipitated DNA was resuspended in $100 \mu \mathrm{L}$ of $\mathrm{H}_{2} \mathrm{O}$.

\section{DNA amplification and array hybridization for ChIP-chip experiments}

DNA amplification was performed using a protocol adapted from Robyr and Grunstein (2003). Briefly, a partially degenerated oligonucleotide (5'-GTTTCCCAGTCACGATCNNNNN NNNN-3') was first incorporated to the immunoprecipitated DNA by the following reaction: $7 \mu \mathrm{L}$ of the immunoprecipitated DNA were incubated with $2 \mu \mathrm{L}$ of $5 \times$ sequenase buffer (USB) and $40 \mathrm{pmol}$ of the degenerated primer, heated for $2 \mathrm{~min}$ at $94^{\circ} \mathrm{C}$, rapidly cooled to $10^{\circ} \mathrm{C}$, and held for $5 \mathrm{~min}$ at $10^{\circ} \mathrm{C}$. Five microliters of a mixture containing $1 \times$ Sequenase Buffer, $0.9 \mathrm{mM}$ $\mathrm{dNTP}, 15 \mathrm{mM}$ DTT, $0.75 \mu \mathrm{g} \mathrm{BSA}$, and $4 \mathrm{U}$ of sequenase 2.0 (USB) were then added to the reaction. The reaction was ramped to $37^{\circ} \mathrm{C}$ at a rate of $0.1^{\circ} \mathrm{C} / \mathrm{sec}$ and then held at $37^{\circ} \mathrm{C}$ for $8 \mathrm{~min}$. The whole process (denaturation, annealing, elongation) is repeated once, with the exception that only $4 \mathrm{U}$ of the sequenase is added after the denaturation step. At the end, the samples were diluted to a final volume of $60 \mu \mathrm{L}$. A simple PCR was then performed on $15 \mu \mathrm{L}$ of the samples, using the fixed sequence of the degenerated oligonucleotide (5'-GTTTCCCAGTCACG ATC-3') and the Taq polymerase (Invitrogen). Amplified DNA was then purified on Qiagen column and eluted in $50 \mu \mathrm{L}$ of water. Five-hundred nanograms of the amplified DNA were then labeled using the random priming labeling kit (Invitrogen), with either $3 \mu \mathrm{L}$ of Cy3dCTP (for the mock ChIP) or Cy5dCTP (for the MSL1 or MSL3 ChIP) (Amersham). The dNTP mixture from the random priming labeling kit was replaced by a mixture containing $1.2 \mathrm{mM}$ dATP, $1.2 \mathrm{mM}$ dTTP, $1.2 \mathrm{mM}$ dGTP, and 0.6 $\mathrm{mM}$ dCTP. Five-hundred nanograms of the two probes were then mixed, purified on Qiagen column, concentrated on MicroconYM-30 (Millipore), dried, and resuspended in $2.8 \mu \mathrm{L}$ of water. The hybridization was performed overnight at $43^{\circ} \mathrm{C}$, in $40 \mu \mathrm{L}$ of hybridization buffer ( $3 \times \mathrm{SSC}, 50 \%$ formamide, $0.8 \mathrm{mg}$ / $\mathrm{mL}_{\text {polyA }}{ }^{+}$[Sigma, catalog no. P9403], $1 \%$ SDS, $5 \times$ Denhardt). The slides were washed once in SDS $0.03 \%, 1 \times$ SSC; once in $0.2 \times$ SSC; and twice in $0.05 \%$ SSC; and centrifuged for $5 \mathrm{~min}$ before scanning.

\section{RNA extraction, amplification, and array hybridization for RNA expression analysis}

RNA was extract from staged dechorionated embryos or from dissected salivary glands, using the RNeasy kit (Qiagen). For the reference sample, RNA from overnight $(0-12$ h) embryos, adults, and salivary glands was mixed at a ratio $1: 1: 1$. One microgram of total RNA was reverse-transcribed and amplified with 13 cycles of PCR, according to the SMART protocol described in Petalidis et al. (2003). The DNA was purified on a Qiagen column prior to labeling. Five-hundred nanograms of this amplified DNA were labeled using the Bioprime DNA Labeling System (InVitrogen) and Cy3 or Cy5-dUTP (Amersham). One microgram of each labeled cDNA was used for hybridization. The hybridization is performed on the cDNA arrays, as described above.

\section{Array design}

For hybridizations we used spotted microarrays containing Drosophila Gene Collection release 1 (DGCr1) (5849 clones) and release 2 (DGCr2) (5061 clones) cDNA fragments, representing $>70 \%$ of the predicted genes in Drosophila (Berkeley Drosophila Genome Project; Stapleton et al. 2002). Clones were amplified by PCR directly from fresh culture. After purification, the PCR products were spotted on Corning Ultra Gaps slides using a Lucidea Array Spotter and cross-linked by applying $300 \mathrm{mV}$ of UV energy.

\section{Real-time PCR analysis}

The primers pairs were designed to amplify 150-200-bp fragments in the middle of the genes, except for the roX1 primers, designed in the previously characterized MSL-binding site.

Real-time PCR analysis of the ChIP was performed using the SYBR Green PCR master mix (Applied Biosystem), $100 \mathrm{ng}$ of each primers, and $4 \mu \mathrm{L}$ of the immunoprecipitated DNA, in an ABI7500 Real-time PCR Instrument (Applied Biosystem). Fold enrichment was determined for each primer according the $\Delta \mathrm{ct}$

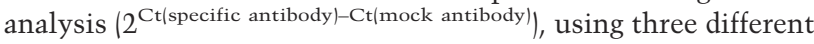
ChIP experiments. For the analysis of the RNA levels, the RNA was first reverse-transcribed using the SuperScript RT (InVitrogen), and $500 \mathrm{ng}$ of random hexamer. One microliter of cDNA was then submitted to real-time PCR using the SYBR Green PCR master mix (Applied Biosystem) and $100 \mathrm{ng}$ of each primer.

\section{Immunofluorescence on polytenes squashes}

Preparation of polytene chromosomes was performed as described (http://www.igh.cnrs.fr/equip/cavalli/Lab\%20Protocols/ Immunostaining.pdf). Rat MSL-1 antibody was used at 1:1000 dilution. S5-P-PolII antibody (H14) was purchased from Covance and used at a 1:50 dilution. Spt5 and Spt6 were a gift from 
Dr J.T. Lis (Cornell University, NY) and both used at a 1:1000 dilution. Images were captured with an AxioCamHR CCD camera on a Zeiss Axiovert200M microscope using a 100× PlanApochomat NA 1.4 oil immersion objective. Alternatively, images were taken using appropriate filter combinations with a Deltavision Spectris optical sectioning microscope (DeltaVision).

\section{Preprocessing, normalization and analysis}

For both expression and enrichment studies, all files were processed and analyzed in the R/Bioconductor programming environment. To remove systematic sources of variation, the data were normalized using the scaled print-tip loess normalization of Yang et al. (2002) within slides and across slides. Both raw and normalized data were visually inspected for outliers and to determine if distributional assumptions were met and if normalization was appropriate. Any spots flagged during visual inspection and gridding were removed from the analysis. When log signal or fold change is reported, it represents the average across all biological replicates (minimum biological replicates was three, maximum was five; see Supplemental Material). For the ChIP-chip experiments median percentile rank was also computed across replicates. A one-sample $t$-test was performed to identify those clones in which enrichment was greater than mock/background (i.e., log ratio $>0$ ). To identify the candidate MSL-1 target population, we used the joint criteria of statistical significance and rank (raw $p$-value $<0.1$ and median percentile rank across the arrays $>95 \%$ ). Given the discovery framework of this study (in which false negatives would have a more detrimental impact, with respect to identification of putative candidates for further follow up, than false positives), raw $p$-values were used in the candidate target criteria for all subsequent analyses, instead of false discovery rate (FDR)-adjusted $p$-values.

For the male and female expression studies, $P$-values were adjusted for multiple comparisons using the Benjamini-Hochberg correction.

For analysis of categorical data, $\chi^{2}$ or Fisher's exact tests were performed as appropriate. In addition to differential gene expression analysis, cluster analysis (average linkage using a correlation metric) was performed as well to aid in visualization (Cluster and Treeview software, http://rana.lbl.gov/EisenSoftware. htm).

\section{Acknowledgments}

We thank J. Luchessi and J. Lis for kindly providing antibodies. We thank L. Steinmetz for critical reading of the manuscript. We are grateful to E. Furlong for help with early stages of the project and V. Benes and EMBL-Genecore facility for help with microarrays. We also thank members of the laboratory for helpful discussions. G.L. is a recipient of EMBO long-term fellowship. M.J.L. acknowledges financial support by the Deutsche Forschungsgemeinschaft. This work was partially supported by FP6EU "Epigenome" and DFG funded SPP1129 "Epigenetics."

\section{References}

Aggarwal, B.D. and Calvi, B.R. 2004. Chromatin regulates origin activity in Drosophila follicle cells. Nature 430: 372-376.

Akhtar, A. and Becker, P.B. 2000. Activation of transcription through histone $\mathrm{H} 4$ acetylation by MOF, an acetyl transferase essential for dosage compensation in Drosophila. Mol. Cell 5: 367-375.

Arbeitman, M.N., Furlong, E.E., Imam, F., Johnson, E., Null, B.H., Baker, B.S., Krasnow, M.A., Scott, M.P., Davis, R.W., and White, K.P. 2002. Gene expression during the life cycle of Drosophila melanogaster. Science 297: 2270-2275.

Baker, B.S., Gorman, M., and Marin, I. 1994. Dosage compensation in Drosophila. Annu. Rev. Genet. 28: 491-521.

Belyakin, S.N., Christophides, G.K., Alekseyenko, A.A., Kriventseva, E.V., Belyaeva, E.S., Nanayev, R.A., Makunin, I.V., Kafatos, F.C., and Zhimulev, I.F. 2005. Genomic analysis of Drosophila chromosome underreplication reveals a link between replication control and transcriptional territories. Proc. Nat1. Acad. Sci. 102: 8269-8274.

Bone, J.R., Lavender, J., Richman, R., Palmer, M.J., Turner, B.M., and Kuroda, M.I. 1994. Acetylated histone H4 on the male X chromosome is associated with dosage compensation in Drosophila. Genes \& Dev. 8: 96-104.

Bourbon, H.M., Gonzy-Treboul, G., Peronnet, F., Alin, M.F., Ardourel, C., Benassayag, C., Cribbs, D., Deutsch, J., Ferrer, P., Haenlin, M., et al. 2002. A P-insertion screen identifying novel X-linked essential genes in Drosophila. Mech. Dev. 110: 71-83.

Bussemaker, H.J., Li, H., and Siggia, E.D. 2001. Regulatory element detection using correlation with expression. Nat. Genet. 27: 167-171.

Chambeyron, S. and Bickmore, W.A. 2004. Chromatin decondensation and nuclear reorganization of the HoxB locus upon induction of transcription. Genes \& Dev. 18: 1119-1130.

Chiang, P.W. and Kurnit, D.M. 2003. Study of dosage compensation in Drosophila. Genetics 165: 1167-1181.

Demakova, O.V., Kotlikova, I.V., Gordadze, P.R., Alekseyenko, A.A., Kuroda, M.I., and Zhimulev, I.F. 2003. The MSL complex levels are critical for its correct targeting to the chromosomes in Drosophila melanogaster. Chromosoma 112: 103-115.

Dion, M.F., Altschuler, S.J., Wu, L.F., and Rando, O.J. 2005. Genomic characterization reveals a simple histone $\mathrm{H} 4$ acetylation code. Proc. Nat1. Acad. Sci. 102: 5501-5506.

Edgar, B.A. and Orr-Weaver, T.L. 2001. Endoreplication cell cycles: More for less. Cell 105: 297-306.

Fagegaltier, D. and Baker, B.S. 2004. X chromosome sites autonomously recruit the dosage compensation complex in Drosophila males. PLoS Biol. 2: e341.

Franke, A., Dernburg, A., Bashaw, G.J., and Baker, B.S. 1996. Evidence that MSL-mediated dosage compensation in Drosophila begins at blastoderm. Development 122: 2751-2760.

Greenberg, A.J., Yanowitz, J.L., and Schedl, P. 2004. The Drosophila GAGA factor is required for dosage compensation in males and for the formation of the male-specific-lethal complex chromatin entry site at 12DE. Genetics 166: 279-289.

Hamada, F.N., Park, P.J., Gordadze, P.R., and Kuroda, M.I. 2005. Global regulation of X chromosomal genes by the MSL complex in Drosophila melanogaster. Genes \& Dev. 19: 22892294.

Henry, R.A., Tews, B., Li, X., and Scott, M.J. 2001. Recruitment of the male-specific lethal (MSL) dosage compensation complex to an autosomally integrated roX chromatin entry site correlates with an increased expression of an adjacent reporter gene in male Drosophila. J. Biol. Chem. 276: 3195331958.

Hilfiker, A., Hilfiker-Kleiner, D., Pannuti, A., and Lucchesi, J.C. 1997. mof, a putative acetyl transferase gene related to the Tip60 and MOZ human genes and to the SAS genes of yeast, is required for dosage compensation in Drosophila. EMBO J. 16: 2054-2060.

Hirose, F., Yamaguchi, M., and Matsukage, A. 1999. Targeted expression of the DNA binding domain of DRE-binding factor, a Drosophila transcription factor, attenuates DNA replication of the salivary gland and eye imaginal disc. Mol. 
Cell. Biol. 19: 6020-6028.

Hochheimer, A., Zhou, S., Zheng, S., Holmes, M.C., and Tjian, R. 2002. TRF2 associates with DREF and directs promoterselective gene expression in Drosophila. Nature 420: 439445.

Jin, Y., Wang, Y., Johansen, J., and Johansen, K.M. 2000. JIL-1, a chromosomal kinase implicated in regulation of chromatin structure, associated with the male specific lethal (MSL) dosage compensation complex. J. Cell Biol. 149: 1005-1010.

Kelley, R.L., Meller, V.H., Gordadze, P.R., Roman, G., Davis, R.L., and Kuroda, M.I. 1999. Epigenetic spreading of the Drosophila dosage compensation complex from roX RNA genes into flanking chromatin. Cell 98: 513-522.

Kotlikova, I.V., Demakova, O.V., Semeshin, V.F., Shloma, V.V., Boldyreva, L.V., Kuroda, M.I., and Zhimulev, I.F. 2006. The Drosophila dosage compensation complex binds to polytene chromosomes independently of developmental changes in transcription. Genetics 172: 963-974.

Kurdistani, S.K., Tavazoie, S., and Grunstein, M. 2004. Mapping global histone acetylation patterns to gene expression. Cell 117: 721-733.

Lucchesi, J.C., Kelly, W.G., and Panning, B. 2005. Chromatin remodeling in dosage compensation. Annu. Rev. Genet. 39: 615-651.

Lyman, L.M., Copps, K., Rastelli, L., Kelley, R.L., and Kuroda, M.I. 1997. Drosophila male-specific lethal-2 protein: Structure/function analysis and dependence on MSL-1 for chromosome association. Genetics 147: 1743-1753.

MacAlpine, D.M., Rodriguez, H.K., and Bell, S.P. 2004 Coordination of replication and transcription along a Drosophila chromosome. Genes \& Dev. 18: 3094-3105.

Mendjan, S., Taipale, M., Kind, J., Holz, H., Gebhardt, P., Schelder, M., Vermeulen, M., Buscaino, A., Duncan, K. Mueller, J., et al. 2006. Nuclear pore components are involved in the transcriptional regulation of dosage compensation in Drosophila. Mol. Cell (in press).

Nowak, S.J. and Corces, V.G. 2004. Phosphorylation of histone H3: A balancing act between chromosome condensation and transcriptional activation. Trends Genet. 20: 214-220.

Oh, H., Park, Y., and Kuroda, M.I. 2003. Local spreading of MSL complexes from roX genes on the Drosophila $\mathrm{X}$ chromosome. Genes \& Dev. 17: 1334-1339.

Orlando, V., Strutt, H., and Paro, R. 1997. Analysis of chromatin structure by in vivo formaldehyde cross-linking. Methods 11: 205-214.

Park, Y., Kelley, R.L., Oh, H., Kuroda, M.I., and Meller, V.H. 2002. Extent of chromatin spreading determined by roX RNA recruitment of MSL proteins. Science 298: 1620-1623.

Petalidis, L., Bhattacharyya, S., Morris, G.A., Collins, V.P., Freeman, T.C., and Lyons, P.A. 2003. Global amplification of mRNA by template-switching PCR: Linearity and application to microarray analysis. Nucleic Acids Res. 31: e142.

Peter, A., Schottler, P., Werner, M., Beinert, N., Dowe, G., Burkert, P., Mourkioti, F., Dentzer, L., He, Y., Deak, P., et al. 2002. Mapping and identification of essential gene functions on the X chromosome of Drosophila. EMBO Rep. 3: 34-38.

Ragoczy, T., Telling, A., Sawado, T., Groudine, M., and Kosak, S.T. 2003. A genetic analysis of chromosome territory looping: Diverse roles for distal regulatory elements. Chromosome Res. 11: 513-525.

Rastelli, L., Richman, R., and Kuroda, M.I. 1995. The dosage compensation regulators MLE, MSL-1 and MSL-2 are interdependent since early embryogenesis in Drosophila. Mech. Dev. 53: 223-233.

Robyr, D. and Grunstein, M. 2003. Genomewide histone acety- lation microarrays. Methods 31: 83-89.

Sass, G.L., Pannuti, A., and Lucchesi, J.C. 2003. Male-specific lethal complex of Drosophila targets activated regions of the $\mathrm{X}$ chromosome for chromatin remodeling. Proc. Natl. Acad. Sci. 100: 8287-8291.

Schubeler, D., Francastel, C., Cimbora, D.M., Reik, A., Martin, D.I., and Groudine, M. 2000. Nuclear localization and histone acetylation: A pathway for chromatin opening and transcriptional activation of the human $\beta$-globin locus. Genes \& Dev. 14: 940-950.

Schubeler, D., Scalzo, D., Kooperberg, C., van Steensel, B., Delrow, J., and Groudine, M. 2002. Genome-wide DNA replication profile for Drosophila melanogaster: A link between transcription and replication timing. Nat. Genet. 32: 438442.

Smith, E.R., Allis, C.D., and Lucchesi, J.C. 2001. Linking global histone acetylation to the transcription enhancement of Xchromosomal genes in Drosophila males. J. Biol. Chem. 276: 31483-31486.

Stapleton, M., Liao, G., Brokstein, P., Hong, L., Carninci, P., Shiraki, T., Hayashizaki, Y., Champe, M., Pacleb, J., Wan, K., et al. 2002. The Drosophila gene collection: Identification of putative full-length cDNAs for $70 \%$ of $D$. melanogaster genes. Genome Res. 12: 1294-1300.

Straub, T., Dahlsveen, I.K., and Becker, P.B. 2005a. Dosage compensation in flies: Mechanism, models, mystery. FEBS Lett. 579: 3258-3263.

Straub, T., Gilfillan, G.D., Maier, V.K., and Becker, P.B. 2005b. The Drosophila MSL complex activates the transcription of target genes. Genes \& Dev. 19: 2284-2288.

Taipale, M. and Akhtar, A. 2005. Chromatin mechanisms in Drosophila dosage compensation. Prog. Mol. Subcell. Biol. 38: $123-149$.

Turner, B.M. 1998. Histone acetylation as an epigenetic determinant of long-term transcriptional competence. Cell. Mol. Life Sci. 54: 21-31.

Turner, B.M., Birley, A.J., and Lavender, J. 1992. Histone H4 isoforms acetylated at specific lysine residues define individual chromosomes and chromatin domains in Drosophila polytene nuclei. Cell 69: 375-384.

van Steensel, B. 2005. Mapping of genetic and epigenetic regulatory networks using microarrays. Nat. Genet. 37 (Suppl.): S18-S24.

Wang, Y., Zhang, W., Jin, Y., Johansen, J., and Johansen, K.M. 2001. The Jil-1 tandem kinase mediates histone H3 phosphorylation and is required for maintenance of chromatin structure in Drosophila. Cell 105: 433-443.

White, E.J., Emanuelsson, O., Scalzo, D., Royce, T., Kosak, S., Oakeley, E.J., Weissman, S., Gerstein, M., Groudine, M., Snyder, M., et al. 2004. DNA replication-timing analysis of human chromosome 22 at high resolution and different developmental states. Proc. Nat1. Acad. Sci. 101: 17771-17776.

Yang, Y.H., Dudoit, S., Luu, P., Lin, D.M., Peng, V., Ngai, J., and Speed, T.P. 2002. Normalization for cDNA microarray data: A robust composite method addressing single and multiple slide systematic variation. Nucleic Acids Res. 30: e15. 


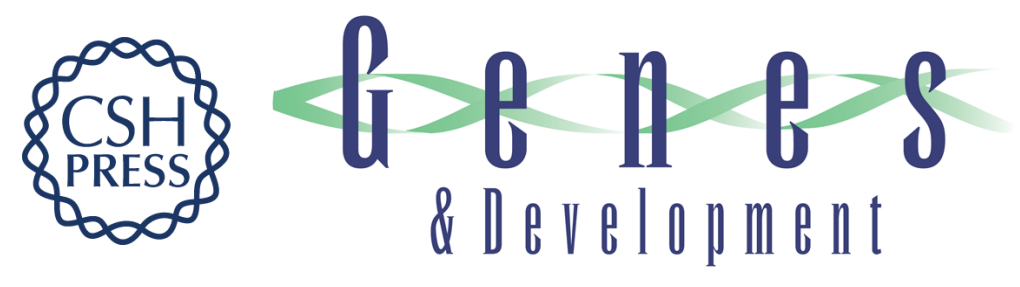

\section{X-chromosome-wide profiling of MSL-1 distribution and dosage compensation in Drosophila}

Gaëlle Legube, Shannon K. McWeeney, Martin J. Lercher, et al.

Genes Dev. 2006, 20:

Access the most recent version at doi:10.1101/gad.377506

\section{Supplemental http://genesdev.cshlp.org/content/suppl/2006/03/16/gad.377506.DC1 \\ Material}

Related Content

Dosage compensation in high resolution: global up-regulation through local recruitment

Dirk Schübeler

Genes Dev. UNKNOWN, 2006 20: 749-753 Chromosome-wide gene-specific targeting of the Drosophila dosage compensation complex

Gregor D. Gilfillan, Tobias Straub, Elzo de Wit, et al.

Genes Dev. UNKNOWN , 2006 20: 858-870 High-resolution ChIPchip analysis reveals that the Drosophila MSL complex selectively identifies active genes on the male $X$ chromosome

Artyom A. Alekseyenko, Erica Larschan, Weil R. Lai, et al.

Genes Dev. UNKNOWN , 2006 20: 848-857

References This article cites 51 articles, 20 of which can be accessed free at:

http://genesdev.cshlp.org/content/20/7/871.full.html\#ref-list-1

Articles cited in:

http://genesdev.cshlp.org/content/20/7/871.full.html\#related-urls

License

Email Alerting Receive free email alerts when new articles cite this article - sign up in the box at the top Service right corner of the article or click here.

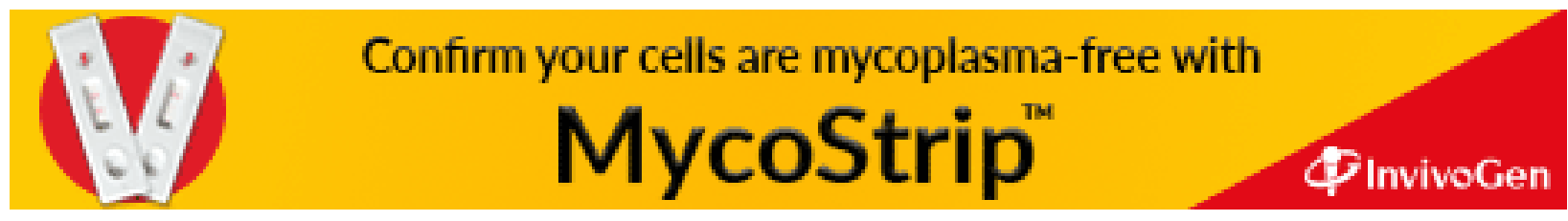

